\title{
Relation entre la vitesse relative de croissance et la teneur en azote chez la laitue (Lactuca sativa $L$ ). Effets de l'ombrage et du niveau de l'alimentation minérale
}

\author{
L Dapoigny ${ }^{1 *}$, P Robin ${ }^{2}$, C Raynal-Lacroix ${ }^{3}$, A Fleury ${ }^{4}$ \\ 1 Chaire des sciences et techniques des productions légumières, ENSH, 2, rue Le Nôtre, F-49000 Angers; \\ 2 Unité de recherche en écophysiologie et horticulture, Inra, domaine Saint-Paul, Agroparc, F-84914 Avignon cedex 9; \\ ${ }^{3}$ Centre expérimental de Lanxade, CTIFL, BP 23, F-24310 La Force; \\ ${ }^{4}$ Laboratoire de recherche sur l'agriculture périurbaine, ENSP, 4, rue Hardy, F-78009 Versailles cedex, France
}

(Reçu le 5 juin 1996; accepté le 23 septembre 1996)

\begin{abstract}
Summary - Study of the relationship between relative growth rate and nitrogen content during lettuce (Lactuca sativa $L$ ) growth: effects of nutrient and radiation levels. The relationship between relative growth rate and total nitrogen content was studied during the vegetative growth of lettuce. Four treatments (two levels of nutrition and two radiation levels) were applied to plants fed with nutrient solution and grown on sand in a plastic greenhouse. On a dry matter basis, the relative growth rate correlates with nitrogen content if we take into account the reduced form of nitrogen only, ie, after the subtraction of nitrate nitrogen from total nitrogen content. The relationship is specific to each treatment; determination coefficients vary between 0.64 and 0.85 . When relative growth rate and nitrogen content are expressed on a fresh matter basis, the correlation is the same for the four treatments and the improved determination coefficients are between 0.92 and 0.95 . These results confirm, in lettuce, the general relationship between nitrogen content and relative growth rate, only when water contribution in biomass and reduced nitrogen form are taken into account.
\end{abstract}

growth / lettuce / nitrogen / nitrate

Résumé - La relation entre la vitesse relative de croissance et la teneur en azote a été étudiée entre le semis et la récolte chez la laitue cultivée sous abri. Quatre traitements : deux niveaux nutritionnels à deux niveaux de rayonnement, ont été appliqués chez des plantes fertirriguées en conditions de hors-sol sur sable. La vitesse relative de croissance exprimée par rapport à la matière sèche n'est corrélée de façon linéaire avec la teneur en azote de cette matière sèche pour chaque traitement que lorsque l'azote est pris en compte sous sa forme réduite après soustraction de l'azote nitrique. Cette relation diffère suivant le traitement, de même que son coefficient de détermination ( $\left.r^{2}\right)$ qui varie de 0,64 à 0,85 . Lorsque la vitesse relative de croissance et la teneur en azote réduit sont exprimées par rapport à la matière fraîche, cette corrélation devient similaire dans les quatre traitements, avec des coefficients de détermination meilleurs compris entre 0,92 et 0,95 . Ces résultats confirment, chez la laitue, l'existence d'une relation générale entre la teneur en azote et la vitesse d'élaboration de la biomasse à condition de prendre en compte la contribution de l'eau dans cette biomasse et l'azote réduit seul.

azote / croissance / laitue / nitrate

* Correspondance et tirés à part

Abréviations : ETP : évapotranspiration potentielle ; MF : matière fraîche ; MS : matière sèche ; Ntot : azote total ; Nréd : azote réduit ; VRC : vitesse relative de croissance. 


\section{INTRODUCTION}

L'accumulation du nitrate chez les végétaux supérieurs peut présenter un caractère spectaculaire et atteindre jusqu'à $4 \%$ de la matière sèche sous forme d'azote nitrique (Maynard et al, 1976). L'étude de son accumulation chez les légumes (Corré et Breimer, 1979) conduit à distinguer cinq groupes dont le plus accumulateur présente fréquemment des teneurs supérieures à $2500 \mathrm{ppm}$ de $\mathrm{NO}_{3}{ }^{-}$par rapport à la matière fraîche. La laitue, l'épinard et le céleri appartiennent à ce groupe. Chez certaines variétés de laitue, l'accumulation de nitrate peut même atteindre 14000 ppm (Corré et Breimer, 1979).

L'accumulation du nitrate dépend notamment du climat et de l'alimentation azotée au cours de croissance de la plante. Chez la laitue comme chez les autres espèces, elle s'observe principalement en hiver. Au printemps et en été, les teneurs en nitrate sont plus faibles mais sont aussi plus sensibles à l'alimentation nitrique (Vermeulen et al, 1987). La fertilisation azotée augmente l'accumulation du nitrate (Eenink et al, 1984 ; Vermeulen et al, 1987 ; Van der Boon et al, 1986) et, à alimentation égale, le rayonnement la diminue (Roorda Van Eysinga et Van Der Meijs, 1985 ; Blom-Zandstra et Lampe, 1985 ; Blom-Zandstra et al, 1988) alors que l'accroissement de la température a, selon les auteurs, des effets contradictoires sur la teneur : diminution (Richard et al, 1985 ; Mayberry et Rauschkolb, 1975), absence d'effet (Richardson et Hardgrave, 1992) ou augmentation (Behr et Wiebe, 1992).

La vitesse relative de croissance d'une plante pendant sa phase exponentielle est en relation étroite avec la teneur en azote total de cette plante (Oscarson et al, 1988, 1989). Au cours du développement, la baisse de la vitesse relative de croissance associée à l'augmentation de la biomasse est aussi concomitante à une diminution de la teneur en azote dans cette biomasse (Caloin et Yu, 1984, 1986 ; Hirose, 1984, 1988 ; Greenwood et al, 1986, 1991). Ces relations entre la vitesse relative de croissance et la teneur en azote ont généralement été établies en prenant en compte d'une part la biomasse sèche, d'autre part l'azote total de la plante (Caloin et $\mathrm{Yu}, 1984,1986$; Oscarson et al, 1988, 1989 ; Ingestad et Agren, 1992 ; Hirose, 1988), y compris chez les plantules de laitues (Burns, 1994a). Cependant, la laitue se distingue parmi les espèces citées en référence, à la fois parce que l'azote nitrique peut être présent en très forte proportion (jusqu'à $40 \%$ de l'azote total), et parce que la teneur en eau est très élevée (jusqu'à $95 \%$ d'eau). On peut alors se demander ce que devient cette relation au cours de la croissance de la laitue, d'autant plus qu'il existe une relation négative entre teneur en nitrate et teneur en matière sèche (Blom-Zandstra et Lampe, 1983, 1985 ; Reinink et al, 1987 ; Behr et Wiebe, 1989 ; Reinink et Blom-Zandstra, 1989).

Pour préciser les relations entre la teneur en azote et la vitesse d'élaboration de la biomasse, deux traitements nutritionnels ont été appliqués à deux niveaux d'éclairement pendant tout le cycle de culture de la laitue. Afin de modifier la teneur en nitrate des plantes sans perturber l'équilibre des anions et des cations, le traitement à faible nutrition est obtenu par dilution du traitement à forte nutrition. Dans ces conditions, l'indicateur de la variable nutritionnelle globale est l'électroconductivité.

\section{MATÉRIEL ET MÉTHODES}

\section{Culture}

Les graines de laitue (Lactuca sativa $L$ variété Judy) sont mises à germer à $20^{\circ} \mathrm{C}$ dans du terreau. Les plantules sont repiquées 13 jours après le semis au stade 4-5 feuilles sur des pots de 3 I (une plante par pot d'une surface de $0,03 \mathrm{~m}^{2}$ ) de sable siliceux inerte (granulométrie entre 0,8 et $1,8 \mathrm{~mm}$ de diamètre). Les plantes sont irriguées avec une solution nutritive à l'aide d'un dispositif "goutte à goutte". Cette solution est préparée par une station automatique de ferti-irrigation (Indal, Holland BV). La fréquence des apports (50 $\mathrm{mL} /$ plante $/ 0,2 \mathrm{~mm}$ d'ETP sous le rayonnement naturel) est contrôlée automatiquement et assure un drainage supérieur à $60 \%$. Cette fréquence est la même pour tous les traitements. Les volumes d'eau apportés chaque jour sur chacun des traitements sont mesurés par des volucompteurs. Une miniparcelle de vingt laitues pour chaque traitement est équipée de gouttières situées sous les pots, afin de mesurer chaque matin le volume de solution nutritive percolé. Le bilan hydrique est calculé par différence entre les volumes apporté et percolé. Les concentrations en $\mathrm{NO}_{3}$ - et les volumes des solutions nutritives apportés et percolés permettent de calculer les quantités d'azote fournies, récupérées et, par différence, consommées.

\section{Traitements}

Deux traitements nutritionnels, N1 faible et N2 fort, sont combinés à deux niveaux de rayonnement, $\mathrm{R} 2$ en éclairement naturel et $\mathrm{R} 1$ en éclairement réduit obtenu par un ombrage à $40 \%$ au moyen d'un filet en poly- 
éthylène vert (AT 1038, Occitania Agri, France). La solution nutritive $\mathrm{N} 2$ contient $1,55 \mathrm{mM}$ de $\mathrm{Ca}\left(\mathrm{NO}_{3}\right)_{2}$, $2,05 \mathrm{mM}$ de $\mathrm{KNO}_{3}, 0,55 \mathrm{mM}$ de $\mathrm{KH}_{2} \mathrm{PO}_{4}, 0,77 \mathrm{mM}$ de $\mathrm{MgSO}_{4}, 1,85 \mathrm{mM}$ de $\mathrm{NaNO}_{3}, 0,99 \mathrm{mM}$ de $\mathrm{NaCl}$. Les oligoéléments complètent la solution nutritive : 15,76 $\mu \mathrm{mol} . \mathrm{L}^{-1}$ de Fe séquestré par le DTPA, 23,46 $\mu \mathrm{mol} . \mathrm{L}^{-1}$ de $\mathrm{Mg}, 8,57 \mu \mathrm{mol} . \mathrm{L}^{-1}$ de $\mathrm{Zn}, 0,8 \mu \mathrm{mol} . \mathrm{L}^{-1}$ de $\mathrm{Cu}$, $63,64 \mu \mathrm{mol} . \mathrm{L}^{-1}$ de B, $0,52 \mu \mathrm{mol} . \mathrm{L}^{-1}$ de Mo. Cette solution N2 se caractérise par une électroconductivité de $960 \mu \mathrm{S} . \mathrm{cm}^{-1}$ et une concentration en $\mathrm{NO}_{3}{ }^{-}$de 7 meq. $\mathrm{L}^{-1}$. La solution nutritive $\mathrm{N} 1$ est obtenue par dilution de la solution N2 de telle sorte que la concentration en $\mathrm{NO}_{3}^{-}$soit de 3 meq. $\mathrm{L}^{-1}$ ce qui conduit à une électroconductivité de $460 \mu \mathrm{S} . \mathrm{cm}^{-1}$. Le pH est maintenu entre 5,7 et 6,1 . Des mesures de rayonnement photosynthétiquement actif sont effectuées au cours de la culture à l'aide de capteurs à l'arséniure de gallium (Pontallier, 1990). Les rayonnements moyens journaliers sont compris en éclairement naturel R2 entre 434 et $170 \mu \mathrm{mol} . \mathrm{m}^{-2} . \mathrm{s}^{-1}$ avec une moyenne de $297 \mu \mathrm{mol}$. $\mathrm{m}^{-2} \cdot \mathrm{s}^{-1}$, et sous ombrage R1 entre 254 et $109 \mu \mathrm{mol}$. $\mathrm{m}^{-2} \cdot \mathrm{s}^{-1}$ avec une moyenne de $181 \mu \mathrm{mol} . \mathrm{m}^{-2} \cdot \mathrm{s}^{-1}$. Les quatre traitements (R1N1, R1N2, R2N1, R2N2) sont appliqués du repiquage à la récolte.

\section{Mesures de biomasse}

Douze prélèvements sont réalisés entre le repiquage et la maturité commerciale à des intervalles variant entre 2 et 7 jours suivant le développement de la culture. À chaque prélèvement et pour chaque traitement, deux lots de quatre plantes sont récoltés au lever du soleil. Les parties aériennes et les racines sont pesées individuellement avant et après séchage ( $48 \mathrm{~h}$ à $80^{\circ} \mathrm{C}$ ). La vitesse relative de croissance est exprimée soit par rapport à la matière sèche soit par rapport à la matière fraîche. Elle est calculée pour chaque période comprise entre deux prélèvements en appliquant la formule (In $\left.P_{2}-\ln P_{1}\right) /\left(t_{2}-t_{1}\right), P_{2}$ et $P_{1}$ étant les masses de matière sèche ou de matière fraîche respectivement aux temps $t_{2}$ et $t_{1}$ (Venus et Causton, 1979). La surface foliaire verte est mesurée pour chaque plante au moyen d'un planimètre (Delta-T Devices Ltd, Cambridge). Pour l'analyse de variance, le programme MGLH, Anova de Systat 5 a été utilisé.

\section{Dosage d'azote}

Les analyses de teneur en azote sont réalisées sur la matière sèche aérienne totale de quatre plantes réunies en un même lot. Des analyses plante par plante sont réalisées sur quatre prélèvements intermédiaires (prélèvement $4,6,9,11$ respectivement pour les jours juliens 227, 233, 240 et 244). L'azote total est mesuré après combustion par un analyseur automatique (LECO SP 428, Michigan, États-Unis) selon la méthode Dumas. Le nitrate est extrait à l'eau et dosé par chromatographie ionique (Dionex Dx 300 , ÉtatsUnis) avec détection conductimétrique. L'azote réduit est calculé par différence entre l'azote total et l'azote nitrique. L'analyse de variance est réalisée pour les quatre prélèvements précités.

Le nitrate de la solution apportée et percolée est analysé par colorimétrie après réduction du nitrate sur une chaîne à cadmium puis réaction avec le sulphanilamide et le $\mathrm{N}$-naphtyléthylène diamine-dichlorure.

\section{RÉSULTATS}

\section{Biomasse et teneur en matière sèche}

L'accumulation de matière fraîche et matière sèche est affectée significativement par la nutrition minérale et par l'éclairement (fig 1). La multi-
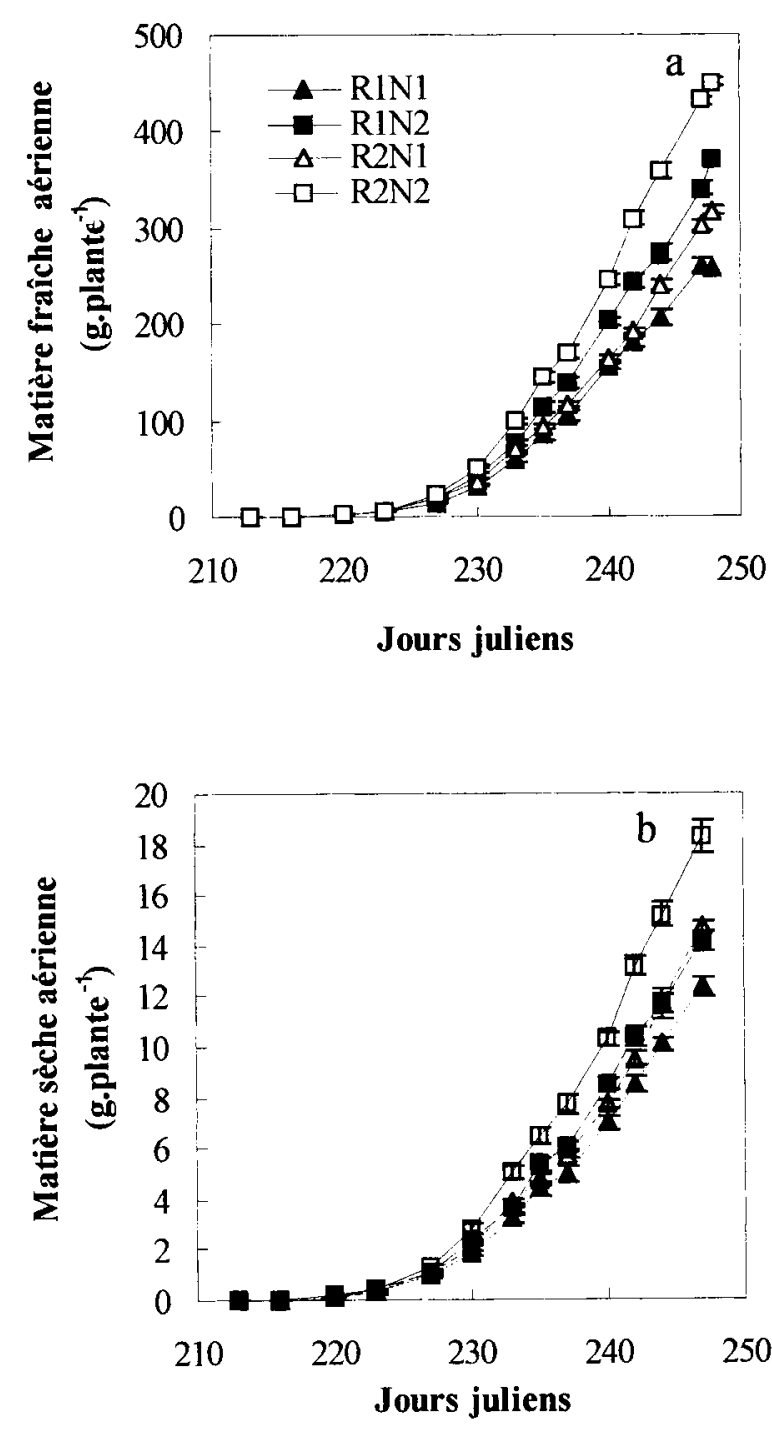

Fig 1. Évolution de la biomasse fraîche aérienne (a) et de la biomasse sèche aérienne (b) de la laitue cultivée sous tunnel en été sur pot avec un éclairement naturel (R2N1 et R2N2) ou avec un éclairement réduit de $40 \%$ (R1N1, R1N2) et alimentée par une solution nutritive de conductivité élevée N2 (R1N2 et R2N2) ou faible N1 (R1N1 et R2N1). Les tirets indiquent l'erreur standard pour l'ensemble des huit plantes récoltées. 
plication par 2,3 de la concentration minérale entre N1 et N2 accroît plus la production de biomasse fraîche que la multiplication du rayonnement par 1,7 entre R1 et R2 (fig 1a). L'effet de la nutrition minérale sur la production de matière fraîche est plus marqué en moyenne sous le rayonnement R2 (+46\%) que sous le rayonnement R1 $(+32 \%)$. Les plantes recevant la nutrition la plus élevée N2 développent aussi une surface foliaire plus importante, en moyenne de $20 \%$, par rapport aux plantes des traitements N1 (résultats non montrés). L'effet du rayonnement sur la production de matière fraîche est plus important en moyenne avec l'alimentation élevée N2 (+28\%) qu'avec l'alimentation faible N1 $(+13 \%)$. Pour la production de biomasse sèche (fig 1b), l'effet de la nutrition minérale est similaire à celui du rayonnement : $+18 \%$ sous $\mathrm{R} 1$ et $+30 \%$ sous R2 pour l'effet de l'accroissement de la nutrition, et $+16 \%$ avec $\mathrm{N} 1$ et $+28 \%$ avec $\mathrm{N} 2$ pour l'effet de l'accroissement du rayonnement. Pour le poids frais comme pour le poids sec, l'effet de la solution nutritive est significatif $(p<$ 0,05 ) dès le deuxième prélèvement (jour julien 220 ), soit 7 jours après la plantation ; l'effet du rayonnement est significatif $(p<0,05)$ à partir du quatrième prélèvement (jour julien 227) après quinze jours de traitements. Une interaction entre les deux facteurs est observable à partir du septième prélèvement (jour julien 235), lequel marque le début de la phase active de pommaison de la laitue.

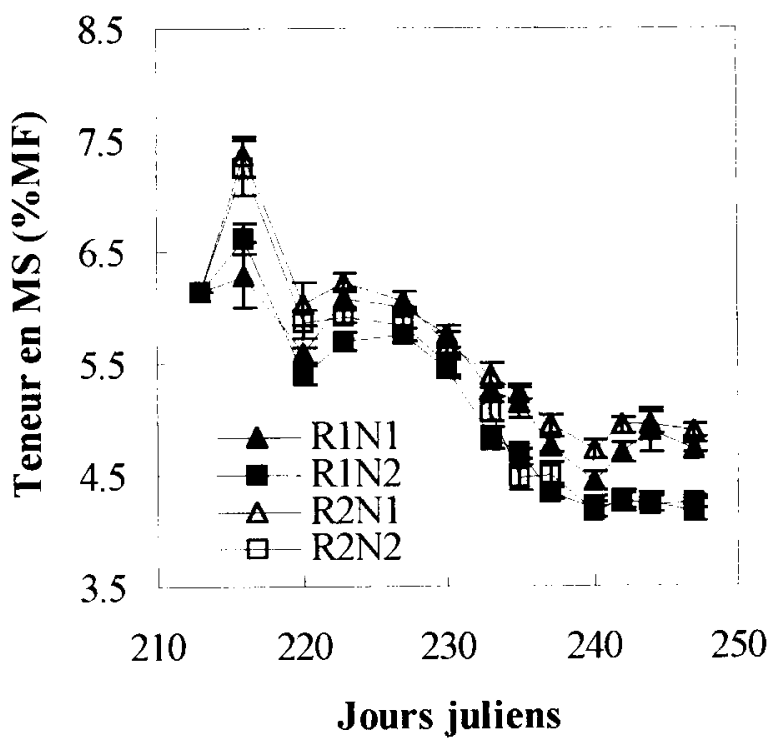

Fig 2. Évolution de la teneur en matière sèche des parties aériennes au cours de la croissance chez la laitue cultivée sous tunnel en été sur pot avec un éclairement naturel (R2N1 et R2N2) ou avec un éclairement réduit de $40 \%$ (R1N1, R1N2) et alimentée par une solution nutritive de conductivité élevée N2 (R1N2 et R2N2) ou faible N1 (R1N1 et R2N1). Les tirets indiquent l'erreur standard $( \pm \mathrm{SE})$.
Au cours du développement de la laitue, la teneur en matière sèche des feuilles baisse de $7 \%$ à $4,5 \%$ (fig 2). Cette teneur est plus élevée en début de culture dans les deux traitements sous éclairement naturel $R 2$ par rapport aux deux traitements ombrés $\mathrm{R} 1(p<0,001)$. La diminution de la teneur en matière sèche en fin de culture est plus accentuée avec la nutrition N2 : les deux traitements R1N2 et R2N2 sont inférieurs de $20 \%$ en moyenne aux traitements R1N1 et R2N1. L'effet de l'alimentation minérale est significatif dès le troisième prélèvement (jour julien 223), huit jours après la plantation. Aucune interaction entre les traitements n'est observée.

\section{Teneurs en azote total, en azote réduit et en nitrate}

Les teneurs en azote total dans la matière sèche (fig 3a) sont nettement supérieures pour le traitement nutritionnel N2 $(p<0,001)$; le traitement ombragé $\mathrm{R} 1$ entraîne une légère augmentation des teneurs pour les deux niveaux de nutrition $(p<0,005$ pour les prélèvements 6,9 et 11). Si les teneurs sont relativement stables pour le traitement N2, une diminution au cours du temps est observée pour N1. Les teneurs en azote réduit dans la matière sèche montrent moins de différences ; cependant les traitements N2 engendrent toujours des teneurs en azote réduit plus élevées que celles des traitements $N 1$ $(p<0,001)$. Le traitement R1N2 présente les teneurs les plus élevées et le traitement R2N1 les teneurs les plus faibles. On observe une diminution des teneurs en azote réduit au cours du temps. Les teneurs en nitrate (fig $3 c$ ) des deux traitements N2, comprises entre 1500 et $2000 \mathrm{mg} \cdot \mathrm{kg}^{-1} \mathrm{MF}$ (unité utilisée pour le critère de qualité chez les légumes), sont deux à trois fois supérieures à celles des traitements $\mathrm{N} 1 \mathrm{com}$ prises entre 500 et $1200 \mathrm{mg} \cdot \mathrm{kg}^{-1} \mathrm{MF}$ $(p<0,001)$. Ces teneurs apparaissent en général plus faibles sous l'éclairage naturel R2 que sous l'ombrage R1; cependant les différences ne sont pas systématiquement significatives. L'analyse plante par plante montre une assez forte variabilité des teneurs en nitrate de l'ordre de $15 \%$.

À chaque prélèvement, on a confronté la teneur en matière sèche de chacun des quatre traitements aux teneurs respectives en nitrate ; on observe systématiquement une relation négative. Pour chacun des cinq derniers prélèvements effectués pendant le stade de pommaison, cette relation présente un coefficient de détermination 

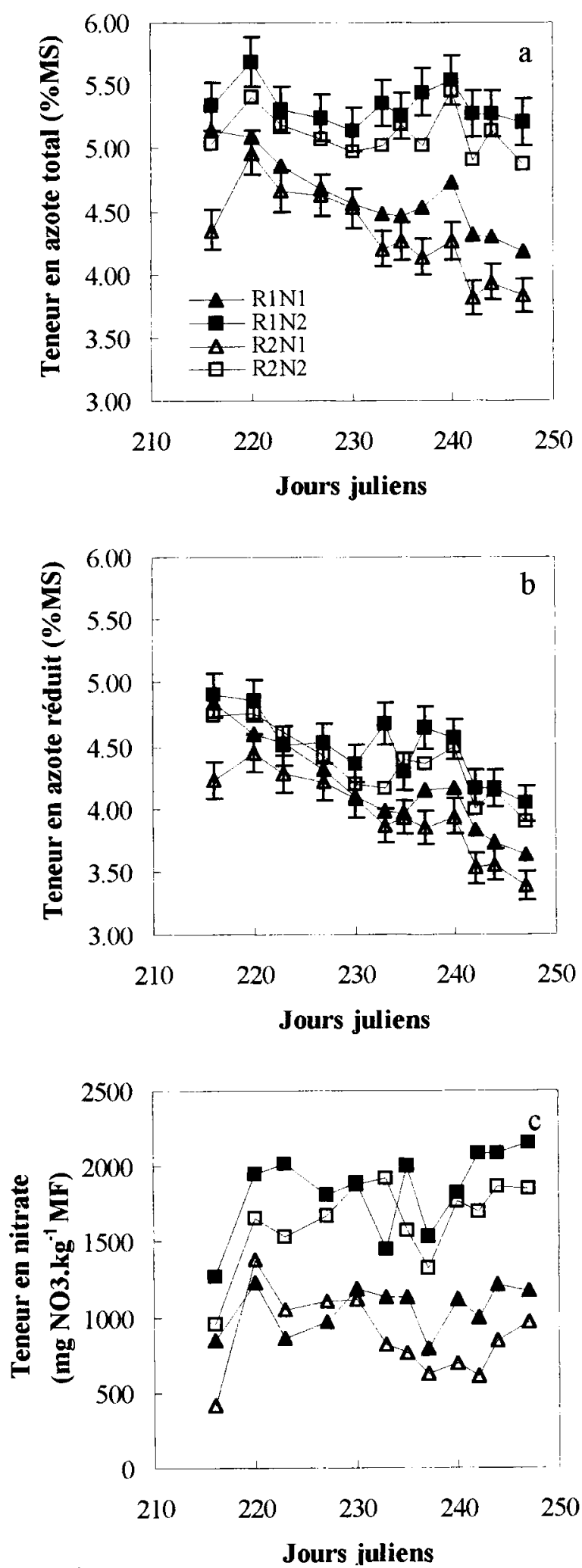

Fig 3. Évolution de la teneur en azote total dans la matière sèche (a), de la teneur en azote réduit dans la matière sèche (b) et de la teneur en nitrate exprimée par rapport à la matière fraîche (c) chez la laitue cultivée sous tunnel en été sur pot avec un éclairement naturel (R2N1 et R2N2) ou avec un éclairement réduit de $40 \%(\mathrm{R} 1 \mathrm{~N} 1, \mathrm{R} 1 \mathrm{~N} 2)$ et alimentée par une solution nutritive de conductivité élevée N2 (R1N2 et R2N2) ou faible N1 (R1N1 et R2N1). En a et b, les tirets indiquent l'erreur standard pour l'ensemble des huit plantes récoltées ; seuls les traitements R1N2 et R2N1 présentent les erreurs afin de ne pas alourdir les figures. variant de 0,92 à 0,99 (fig 4).

Le pourcentage de l'azote percolé par rapport à l'azote apporté est supérieur à $70 \%$ en début de culture et baisse rapidement pendant les deux premières semaines (fig 5). II se maintient ensuite à une valeur moyenne de l'ordre de 50 et $40 \%$ respectivement pour les traitements $\mathrm{R} 1 \mathrm{~N} 2$ et R2N2 et de l'ordre de 25 à $10 \%$ pour les traitements R1N1 et R2N1.

\section{Vitesse relative de croissance et teneur en azote}

Dans le traitement R1N2 (fig 6) qui accumule le plus de nitrate, la vitesse relative de croissance exprimée par rapport à la matière sèche est presque indépendante $\left(r^{2}=0,11\right)$ de la teneur en azote total exprimée par rapport à cette matière sèche (fig 6a) ; la relation s'affirme $\left(r^{2}=0,68\right)$ quand l'azote nitrique est soustrait de l'azote total pour ne prendre en compte que l'azote réduit (fig 6b). L'expression des teneurs en azote par rapport à la matière fraîche améliore encore ces relations : les coefficients de détermination passent de 0,11 à 0,86 pour l'azote total, et de 0,68 à 0,85 pour l'azote réduit (fig $6 \mathrm{c}$ et d). Lorsque la vitesse relative de croissance est calculée à partir de la matière fraîche, les relations obtenues avec les teneurs en azote total et en azote réduit, exprimées par rapport à cette matière fraîche, ont un coefficient identique de 0,95 (fig $6 \mathrm{e}$ et f).

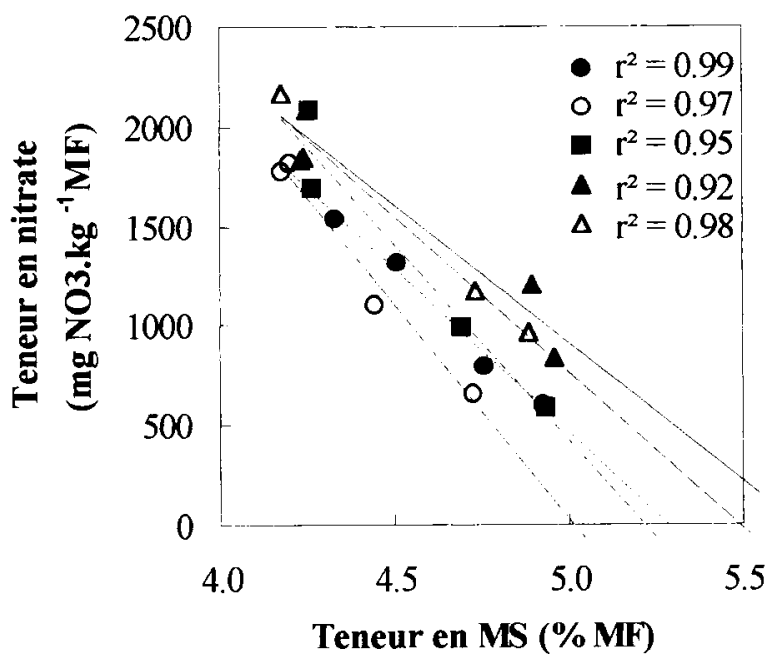

Fig 4. Relations pour les cinq dernières dates de récolte effectuées les jours juliens 237 (rond noir), 240 (rond blanc), 242 (carré noir), 244 (triangle noir), 247 (triangle blanc) entre la teneur en nitrate et la teneur en matière sèche pour la laitue cultivée sous tunnel en été sur pot avec un éclairement naturel ou avec un éclairement réduit de $40 \%$ et alimentée par une solution nutritive de conductivité élevée ou faible. 
L'amélioration des relations entre la vitesse relative de croissance et les teneurs en azote total et réduit, consécutive à l'expression de ces paramètres par rapport à la matière fraîche, est observée dans chacun des quatre traitements (fig 7). Lorsque la vitesse relative de croissance et les teneurs en azote sont exprimées par rapport à la matière sèche, les quatre relations sont très différentes et plus ou moins bien déterminées (fig $7 \mathrm{a}$ et $\mathrm{b}$ ). Elles sont améliorées par l'expression des teneurs en azote dans la matière fraîche (fig 7c et d). Avec l'azote total ces relations restent différentes selon les traitements (fig 7c) alors qu'avec l'azote réduit ces relations deviennent très voisines avec des coefficients de détermination variant de 0,84 à 0,97 (fig $7 d$ ). Enfin, lorsque la vitesse relative et les teneurs sont exprimées par rapport à la matière fraîche, les relations sont toujours différentes avec l'azote total (fig 7e) alors qu'elles sont identiques avec l'azote réduit (fig $7 f$ ). Dans ce dernier cas, les coefficients de détermination sont compris entre 0,92 et 0,95 selon les traitements.

\section{DISCUSSION}

\section{Conditions de culture}

Dans le protocole retenu, les différents états nutritionnels sont obtenus par une modification des concentrations en ions minéraux dans la

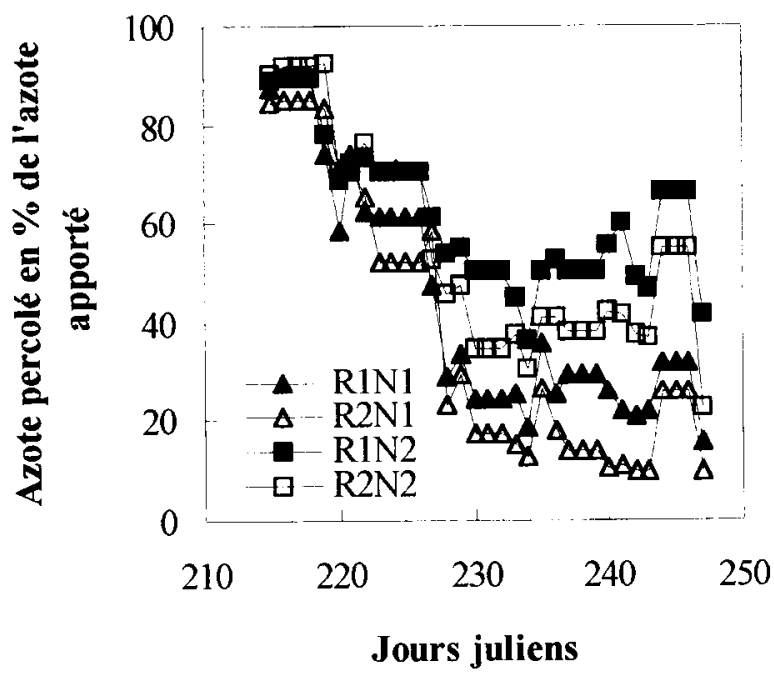

Fig 5. Évolution journalière de la quantité d'azote percolé exprimée en pourcentage de l'azote apporté à un lot de 20 laitues cultivées sous tunnel en été sur pot avec un éclairement naturel (R2N1 et R2N2) ou avec un éclairement réduit de $40 \%(\mathrm{R} 1 \mathrm{~N} 1, \mathrm{R} 1 \mathrm{~N} 2)$ et alimentée par une solution nutritive de conductivité élevée N2 (R1N2 et R2N2) ou faible N1 (R1N1 et R2N1). solution nutritive qui sont 2,3 fois plus concentrés en N2 qu'en N1. Dans notre dispositif sur substrat, le système d'irrigation fonctionne par intermittence et les racines modifient en permanence les concentrations des ions, lesquelles sont donc variables dans le temps et hétérogènes au sein du support. La conductivité et les concentrations des solutions d'apport sont donc des indicateurs approximatifs pour caractériser l'environnement racinaire. Ainsi dans nos conditions expérimentales, une solution à $460 \mu \mathrm{S} . \mathrm{cm}^{-1}$ avec une concentration en nitrate de 3 meq. $L^{-1}$ est encore limitante de la croissance alors qu'en hydroponie stricte avec une concentration en nitrate maintenue à 0,36 meq. $L^{-1}$ une culture d'hiver de laitue présente une croissance optimale (Letey et al, 1982). La nutrition dépend en fait du nombre d'irrigations et donc des conditions climatiques

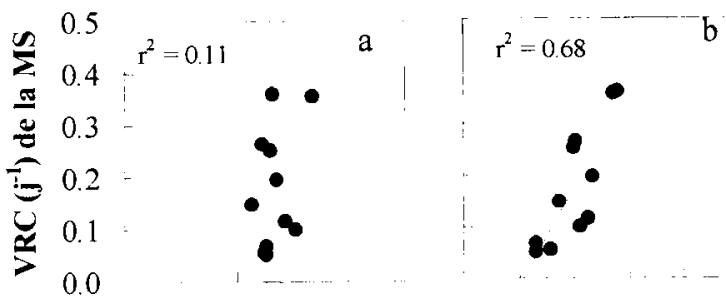

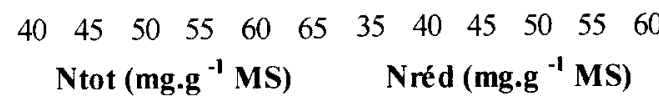
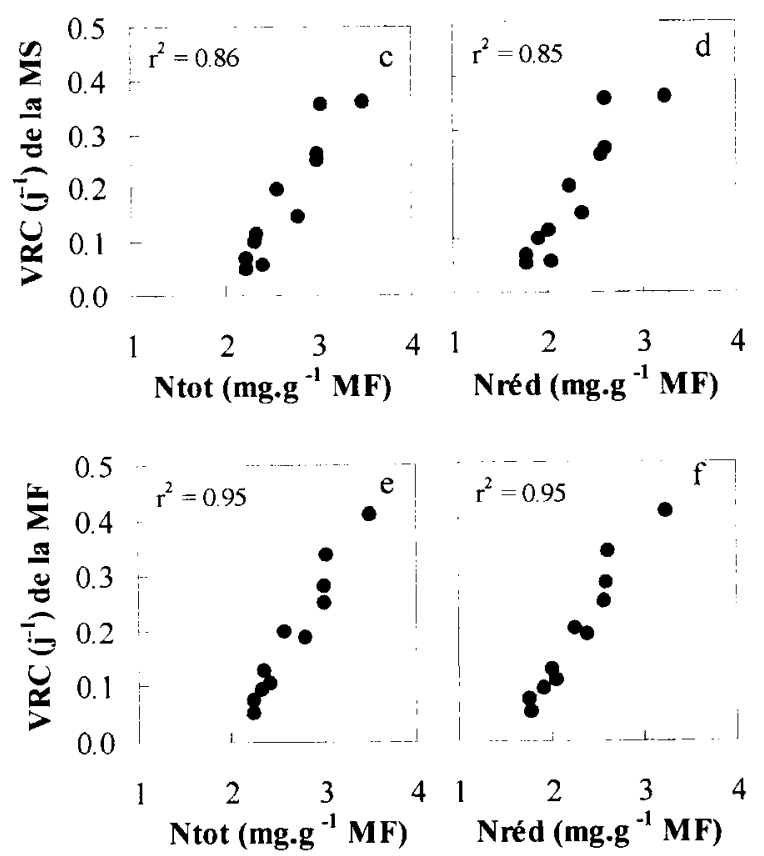

Fig 6. Relations au cours de la culture entre la vitesse relative de croissance de la matière sèche $(\mathbf{a}, \mathbf{b}, \mathbf{c}, \mathbf{d})$ ou en matière fraîche $(\mathbf{e}, \mathbf{f})$ et la teneur en azote total $(\mathbf{a}, \mathbf{c}, \mathbf{e})$ ou en azote réduit $(\mathbf{b}, \mathbf{d}, \mathbf{f})$ exprimée par rapport à la matière sèche (a, b) ou à la matière fraîche $(\mathbf{c}, \mathbf{d}, \mathbf{e}, \mathbf{f})$ chez la laitue cultivée sous tunnel en été sur pot avec un éclairement naturel réduit de $40 \%$ et alimentée par une solution nutritive de conductivité élevée (R1N2). 
extérieures et de l'état de la plante. Une fréquence réduite des apports de solution nutritive pourra conduire à un épuisement des ions, et du nitrate en particulier, dans l'environnement racinaire et à une diminution importante de leur concentration dans le percolat. Si le nitrate apporté n'est pas limitant il y aura une forte proportion de cet ion dans le percolat. La proportion d'azote percolé par rapport à l'azote apporté est donc un indicateur du niveau de satisfaction de l'offre par rapport à la demande en azote. Les plantes cultivées avec une alimentation $\mathrm{N} 1$ sous ombrage R1 sont donc limitées dans leur croissance (fig 5), puisque le pourcentage de nitrate percolé devient systématiquement inférieur à $30 \%$ des apports dès que les plantes ont atteint $20 \mathrm{~g}$ de matière fraîche. Les plantes sous rayonnement naturel R2 présentent un pourcentage de nitrate percolé encore plus faible dû à une consommation plus grande de nitrate par rapport aux plantes ombrées R1. Chez les plantes recevant la nutrition N2 sous rayonnement naturel R2 le pourcentage d'azote percolé est inférieur à $40 \%$ des apports. Ces observations sur les per-
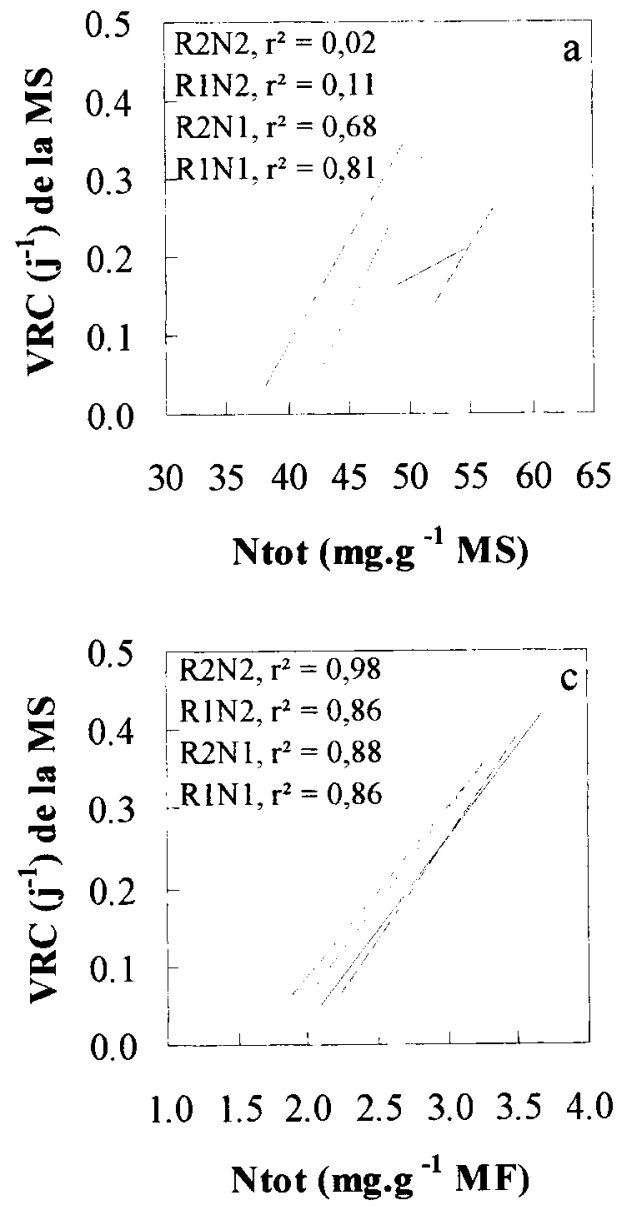
culture entre la vitesse relative de croissance relative de la matière sèche $(\mathbf{a}, \mathbf{b}, \mathbf{c}, \mathbf{d})$ ou de la matière fraîche $(e, f)$ et la teneur en azote total $(\mathbf{a}, \mathbf{c}, \mathbf{e})$ ou en azote réduit (b, $\mathbf{d}, \mathbf{f})$ exprimées par rapport à la matière sèche $(\mathbf{a}, \mathbf{b})$ ou à la matière fraîche $(\mathbf{c}, \mathbf{d}, \mathbf{e}, \mathbf{f})$ chez la laitue cultivée avec un éclairement naturel $\mathrm{R} 2$ et une solution nutritive à conductivité élevée N2 (R2N2, -) ou faible (R2N1, •-•-•) ou avec un éclairement réduit de $40 \%$ et une solution à conductivité élevée (R1N2, - - -) ou réduite (R1N1, ...). Les $r^{2}$ inclus dans chaque figure indiquent les coefficients de détermination des droites de régression. $\begin{array}{llllllll}30 & 35 & 40 & 45 & 50 & 55 & 60 & 65\end{array}$ Ntot (mg.g ${ }^{-1}$ MS)
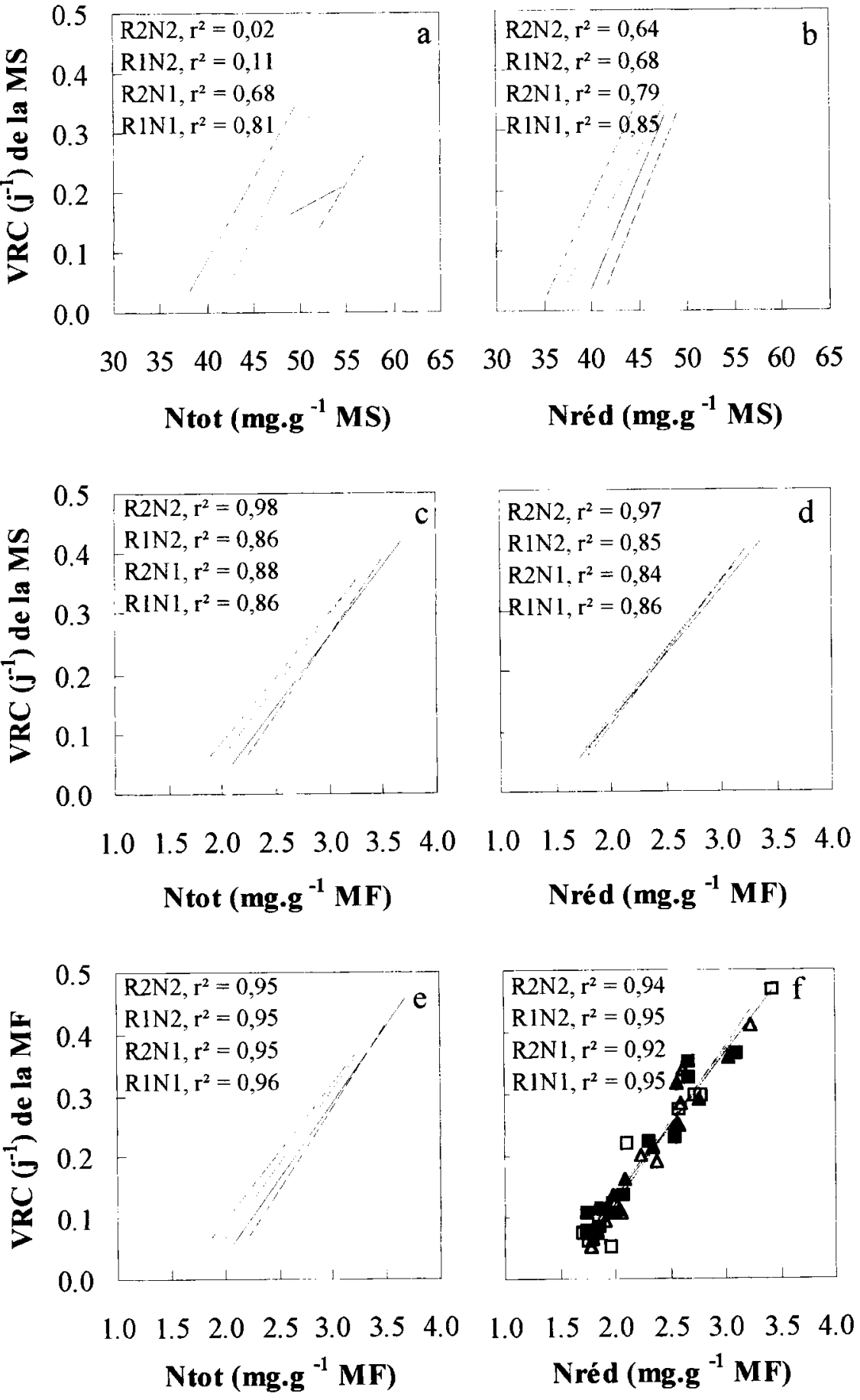
colats suggèrent une consommation minérale importante et n'excluent pas la possibilité d'une limitation de la croissance par la disponibilité minérale. Ce sont les plantes soumises au traitement ombré R1 avec la solution N2 qui présentent le pourcentage d'azote percolé le plus important, indiquant des conditions d'alimentation minérales les plus proches de l'optimum. II est d'ailleurs significatif que le traitement le plus proche de l'optimum, R1N2, présente les teneurs en azote réduit les plus fortes, et le traitement le plus limitant, R2N1, les teneurs les plus faibles. Malgré ces difficultés de caractérisation, les quatre traitements génèrent des différences de croissance (fig 1), des teneurs en matière sèche (fig 2) et en azote (fig 3) qui permettent d'analyser les relations entre les vitesses relatives de croissance et ces teneurs en azote au cours de la croissance de la laitue.

\section{Vitesse relative de croissance et teneur en azote par rapport à la matière sèche}

Les études antérieures portant sur la relation entre vitesse relative de croissance et teneur en azote ont généralement été réalisées en prenant en compte l'azote total de la plante (Caloin et $\mathrm{Yu}$, 1984, 1986 ; Hirose, 1984, 1988 ; Oscarson et al, 1989 ; Greenwood et al, 1986, 1991). Cette prise en compte de l'azote total peut se justifier quand les conditions expérimentales et les espèces étudiées ne conduisent pas à une accumulation importante de nitrate. Ceci n'est plus vrai lorsque celui-ci constitue une part importante dans l'azote total. Or, dans nos conditions expérimentales, l'accumulation du nitrate s'observe même chez les plantes où les conditions de nutrition N1 limitent la croissance, soulignant la forte capacité d'accumulation du nitrate chez la laitue (Corré et Breimer, 1979). La soustraction de l'azote nitrique de l'azote total pour ne prendre en compte que l'azote réduit accumulé dans la plante, améliore la relation entre teneur en azote et vitesse relative de croissance de la matière sèche. Cette observation est analogue à celle faite chez le blé pour déterminer la courbe critique en azote qui caractérise l'évolution de la teneur en azote en fonction de la production de biomasse au cours du développement (Justes et al, 1994). En effet, la relation entre cette teneur en azote nitrique, en dessous de laquelle il y a réduction de la croissance, et la biomasse est améliorée lorsque l'azote nitrique est soustrait de l'azote total. Le nitrate accumulé apparaît donc comme un surplus d'azote sans effet sur la vites- se relative de croissance. Aucune relation n'est d'ailleurs observée entre cette vitesse et la teneur en nitrate dans nos différents traitements (résultats non montrés). Le nitrate, présent principalement dans les vacuoles (Ferrari et al, 1973 ; Grandstedt et Huffaker, 1982), n'intervient pas dans l'activité métabolique autrement que par la fourniture de substrat à la voie de réduction en fonction de la demande de la plante. En revanche une grande partie de l'azote réduit (50 à $70 \%$ ), investi principalement dans les complexes photosynthétiques et enzymatiques, participe directement à la fixation du $\mathrm{CO}_{2}$ (Hunt et al, 1985 ; Evans, 1989, 1990). C'est ainsi qu'une relation linéaire entre la teneur en azote et la teneur en chlorophylle est observée, ainsi qu'entre la teneur en azote réduit et l'activité de la RuBP carboxylase (Evans, 1989). La participation active de l'azote réduit à l'élaboration de la biomasse de la plante explique donc sa relation étroite avec la vitesse relative de croissance (Freijsen et Veen, 1990) qui est observée dans ces résultats. Des travaux précédents sur laitue (Burns, 1994b) montrent une relation curvilinéaire entre la VRC et la teneur en azote réduit de la plante ; cependant ils ont été réalisés dans un contexte différent puisqu'il s'agit de très jeunes plantes $(<2 \mathrm{~g}$ de MF) ayant été carencées en nitrate. II s'agit donc de conditions de croissance relativement différentes, où la plante ne dispose que de son stock d'azote pour assurer sa croissance ; toute comparaison s'avère de fait difficile. Ces deux relations (linéaire et curvilinéaire) pourraient montrer, comme le propose Burns, deux fonctionnements distincts de la plante selon un apport endogène ou exogène d'azote (Burns, 1994a).

\section{Expression des teneurs en azote par rapport à la matière fraîche}

L'amélioration des coefficients de détermination obtenue lorsque les teneurs en azote sont exprimées par rapport à la matière fraîche (fig $7 \mathrm{c}$ et d) rejoint des observations effectuées chez le blé (Leigh et Johnson, 1985). En ne considérant que l'azote réduit, cette amélioration se traduit par un regroupement des quatre traitements. En fait, l'expression de la teneur en azote par rapport à la matière fraîche prend en compte les différences induites par les traitements sur cette teneur en matière sèche (fig 2), et traduit le rôle conjoint de l'azote et de l'eau dans le contrôle de l'élaboration de la matière sèche. Ce point doit être souligné d'autant plus que l'eau participe, 
entre autres, à l'extension cellulaire et favorise ainsi la formation de biomasse (Boyer, 1988).

En fin de culture, la comparaison des différents traitements à différentes dates (fig 4) confirme la relation négative entre teneur en nitrate et teneur en matière sèche (ou positive avec la teneur en eau) observée par d'autres auteurs (Reinink et al, 1987). Cette relation négative s'observe entre différentes variétés de laitue, où l'on constate que les fortes teneurs en matière sèche sont à la fois associées à de fortes teneurs en composés organiques dans la vacuole (Reinink et Blom-Zandstra, 1989) et à de faibles teneurs en nitrate. La diminution du nitrate est alors interprétée comme une participation accrue des sucres et des anions au maintien de la pression osmotique de la vacuole. Cette relation négative entre teneur en nitrate et teneur en sucres ou en anions organiques s'observe aussi bien en comparant différentes variétés de laitue (Berh et Wiebe, 1989, 1992) que chez une même variété soumise à différentes conditions d'alimentation minérale (Blom-Zandstra et Lampe, 1983) ou d'éclairement (Blom-Zandstra et Lampe, 1985). La baisse de rayonnement qui diminue la synthèse des sucres et des acides organiques, et donc leur disponibilité pour le maintien de la pression osmotique, diminue aussi la réduction du nitrate, ce qui favorise son accumulation. Ces résultats soulignent la fonction d'osmoticum assurée par le nitrate, qui se substitue aux composés organiques solubles lorsque l'accumulation de ceux-ci est réduite. C'est ainsi que dans notre expérimentation, les teneurs en nitrate des plantes cultivées sous de faibles éclairements sont supérieures à celles des plantes obtenues sous de forts éclairements pour les deux traitements azotés (fig $3 c$ ).

Cependant, cette augmentation de teneur est surtout marquée, quel que soit le rayonnement, par le passage d'une nutrition limitante (à faible conductivité) à une nutrition proche de l'optimum (à forte conductivité). Chez le coton soumis à une nutrition azotée limitante, les mouvements d'eau sont altérés au sein de la plante qui présente alors des caractéristiques morphologiques identiques à celles d'une plante soumise à un déficit hydrique (Radin et Parker, 1979). Si une nutrition nitrique optimale favorise la synthèse protéique et la multiplication cellulaire (Lawlor et al, 1988), elle agit également sur l'élongation cellulaire, soit par une modification de la conductivité hydraulique de la plante (Radin et Boyer, 1982 ; Barthes et al, 1995), soit par une modification de la propriété des parois cellulaires (Palmer et al, 1996). Chez les plantes déficientes en azote, une réduction de l'expansion cellulaire est observée. La teneur en eau de la plante apparaît donc étroitement liée aux conditions d'alimentation nitrique, lesquelles favorisent le grandissement cellulaire et par voie de conséquence l'accumulation d'eau. Bien que dans nos conditions expérimentales, ce soit le niveau général de l'alimentation minérale qui diminue, et non la fourniture en nitrate seul, ces résultats sur la laitue vont dans le sens des observations précitées. L'augmentation de la taille des cellules est associée, chez la laitue, à l'élévation de la teneur en nitrate et à l'augmentation de la teneur en eau (Reinink et Blom-Zandstra, 1989). Cette observation se retrouve au sein de la plante en fonction de l'âge des feuilles: les feuilles vertes les plus externes qui ont terminé leur croissance ont de grandes cellules (Bensink, 1971) et une teneur en nitrate plus élevée que les jeunes feuilles du cœur de la pomme, à l'abri de la lumière et dont les cellules de petite taille n'ont pas terminé leur croissance et présentent de faibles teneurs en nitrate (Corré et Breimer, 1979 ; Schulze et al, 1985). Nos observations, qui associent croissan$c e$, teneur en eau et teneur en nitrate, permettent de comprendre l'absence de corrélation lorsque la teneur en azote total est rapportée à la matière sèche (fig $7 a$ ) et l'existence de corrélations dont les coefficients de détermination sont proches de 0,9 lorsque cette teneur est rapportée à la matière fraîche (fig $7 \mathrm{c}$ ).

La relation entre la vitesse relative de croissance et la teneur en azote, en particulier en azote réduit, est améliorée lorsque ces deux paramètres sont exprimés par rapport à la matière fraîche (fig 7f). Cela confirme bien la part de l'eau dans la croissance en matière fraîche (Boyer, 1988). La limitation de l'alimentation minérale conduit à une baisse de la teneur en eau et par voie de conséquence de la croissance en poids frais.

\section{CONCLUSION}

En conclusion, la corrélation généralement observée (Ingestad et Agren, 1992 ; Greenwood et al, 1991) entre la vitesse relative de croissance et la teneur en azote dans la matière sèche ne peut être vérifiée chez la laitue, espèce fortement accumulatrice de nitrate, que si l'azote nitrique est soustrait de l'azote total. Le nitrate n'intervient donc pas de façon active dans l'expression de la croissance lorsque celle-ci est réduite à la seule matière sèche et seul l'azote réduit doit être pris 
en compte. Cependant le nitrate accumulé est un agent osmotique qui participe au contrôle du statut hydrique de la plante. C'est pourquoi l'expression de l'azote par rapport à la matière fraîche qui permet la prise en compte de cette association, améliore les relations obtenues avec la vitesse relative de croissance en matière sèche, et encore plus en matière fraîche. Quels que soient les traitements, en termes de niveau de nutrition minérale ou de flux de rayonnement, c'est l'expression de l'azote sous sa seule forme réduite dans la biomasse de matière fraîche, qui permet d'unifier l'ensemble des résultats obtenus, et de simplifier la modélisation générale de la croissance.

\section{REMERCIEMENTS}

Nous remercions le CTIFL d'avoir accueilli le dispositif expérimental sur son centre de Lanxade à Bergerac, ainsi que $\mathrm{J}$ Marchal (Cirad-Flhor) pour le dosage de l'azote total et du nitrate des échantillons. Ce travail a été financé par le ministère de l'Agriculture (DGER) dans le cadre de l'action "Formation par la recherche».

\section{RÉFÉRENCES}

Barthes L, Bousser A, Hoarau J, Deléens E (1995) Reassessment of the relationship between nitrogen supply and xylem exudation in detopped maize seedlings. Plant Physiology Biochem 33, 173-183

Bensink J (1971) On morphogenesis of lettuce leaves in relation to light and temperature. Meded Landbouwhogesch Wageningen Nederland, $93 \mathrm{p}$

Behr U, Wiebe HJ (1989) Nitrate content and osmotically active components in lettuce (Lactuca sativa $\mathrm{L}$ var Capitata L) cultivars. Acta Hortic 244, 99-105

Behr U, Wiebe HJ (1992) Relation between photosynthesis and nitrate content of lettuce cultivars. Scientia Hortic 49, 175-179

Blom-Zandstra G, Lampe JEM (1983) The effect of chloride and sulphate salts on the nitrate content in lettuce plants (Lactuca sativa L). J Plant Nutr 6 , 611-628

Blom-Zandstra M, Lampe JEM (1985) The role of nitrate in the osmoregulation of lettuce (Lactuca sativa $L$ ) grown at different light intensities. J Exp Bot 36, 1043-1052

Blom-Zandstra M, Lampe JEM, Ammerlaan HM (1988) $\mathrm{C}$ and $\mathrm{N}$ utilisation of two lettuce genotypes during growth under non-varying light conditions and after changing the light intensity. Physiol Plant 74, 147153

Boyer JS (1988) Cell enlargement and growth-induced water potentials. Physiol Plant 73, 311-316
Burns IG (1994a) Studies of the relationship between the growth rate of young plants and their total-N concentration using nutrient interruption techniques: theory and experiments. Ann Bot 74, 143-157

Burns IG (1944b) A mechanistic theory for the relationship between growth rate and the concentration of nitrate- $\mathrm{N}$ or organic- $\mathrm{N}$ in young plants derived from nutrient interruption experiments. Ann Bot 74, 159 172

Caloin M, Yu O (1984) Analysis of time course of change in nitrogen content in Dactylis glomerata $\mathrm{L}$ using a model of plant growth. Ann Bot 54, 69-76

Caloin M, Yu O (1986) Relation entre dilution de l'azote et cinétique de croissance chez les graminées. agronomie 6, 167-174

Corré WJ, Breimer T (1979) Nitrate and Nitrite in Vegetables. Pudoc, Wageningen, Pays-Bas

Eenink $\mathrm{AH}$, Blom-Zansdtra $\mathrm{M}$, Hollman $\mathrm{PCH}$, Aarts $\mathrm{P}$, Groenwold R (1984) Research on reduction of nitrate content in lettuce via breeding. In : Legumes à feuilles, Colloque Eucarpia, Versailles, 28-29 fév 1984, 100-109

Evans JR (1989) Photosynthesis and nitrogen relationships in leaves of $C_{3}$ plants. Oecologia 78, 9-19

Evans JR (1990) Photosynthesis: the dependence on nitrogen partitioning. In : Causes and Consequences of Variation in Growth Rate and Productivity of Higher Plants (H Lambres, ML Cambridge, $\mathrm{H}$ Konings, RL Pons, eds), SPB Academic Publishing, La Haye, Pays Bas

Ferrari T, Yoder OC, Filner P (1973) Anaerobic nitrite production by plant cells and tissues: evidence for two nitrate pools. Plant Physiol 51, 423-431

Freijsen AHJ, Veen BW (1990) Phenotypic variation in growth as affected by $\mathrm{N}$-supply: nitrogen productivity. In : Causes and Consequences of Variation in Growth Rate and Productivity of Higher Plants $(\mathrm{H}$ Lambres, ML Cambridge, $H$ Konings, TL Pons, eds), SPB Academic Publishing, La Haye, PaysBas, 19-33

Grandsted R, Huffaker RC (1982) Identification of the leaf vacuole as a major nitrate storage pool. Plant Physiol 70, 410-413

Greenwood DJ, Neeteson JJ, Draycott A (1986) Quantitative relationships for the dependence of growth rate of arable crops on the nitrogen content, dry weight and aerial environment. In : Fundamental, Ecological and Agricultural Aspects of Nitrogen Metabolism in Higher Plants $(\mathrm{H}$ Lambers, JJ Neeteson, I Stulen, eds), Martinius Nijhoff, Boston, MA, États-Unis, 367-387

Greenwood DJ, Gastal F, Lemaire G, Draycott A, Millard P, Neeteson JJ (1991) Growth rate and \%N of field grown crops: theory and experiments. Ann Bot 67, 181-190

Hirose T (1984) Nitrogen use efficiency in growth of Polygonum cuspidatum Sieb et Zuc. Ann Bot 54, 695-704 
Hirose T (1988) Modelling the relative growth rate as a function of plant nitrogen concentration. Physiol Plant 72, 185-189

Hunt ER, Weber JA, Gates DM (1985) Effects of nitrate application on Amaranthus powellii Wats. III. Optimal allocation of leaf nitrogen for photosynthesis and stomatal conductance. Plant Physiol 79, 619-624

Ingestad T, Agren G (1992) Theories and methods on plant nutrition and growth. Physiol Plant 84, 177184

Justes E, Mary B, Meynard JM, Machet JM, ThellierHuche $L$ (1994) Determination of a critical nitrogen dilution curve for winter wheat crops. Ann Bot 74, 397-407

Lawlor DW, Boyle FA, Keys AJ, Kendall AC, Young AT (1988) Nitrate nutrition and temperature effects on wheat: a synthesis of plant growth and nitrogen uptake in relation to metabolic and physiological processes. J Exp Bot 39, 329-343

Leigh RA, Johnson AE (1985) Nitrogen concentrations in field-grown spring barley: an examination of the usefulness of expressing concentrations on the basis of tissue water. J Agric Sci Camb 105, 397406

Lemaire G, Salette J (1984) Relation entre dynamique de croissance et dynamique de prélèvement d'azote pour un peuplement de graminées fourragères. I. Étude de l'effet du milieu. agronomie 4, 423-430

Letey J, Jarrel WM, Valoras N (1982) Nitrogen and water uptake patterns and growth of plants at various minimum solution nitrate concentrations. $J$ Plant Nutr 5, 73-89

Mayberry KS, Rauschkolb RS (1975) Nitrogen uptake in midwinter lettuce. California Agriculture 3, 6-7

Maynard DN, Barker AV, Minotti PL, Peck NH (1976) Nitrate accumulation in vegetable. Adv Agron 28, 71-117

Oscarson P, Ingermarson B, Ugglas MAF, Larsson CM (1988) Characterisation of $\mathrm{NO}_{3}$-uptake in Lemna and Pisum. Plant Soil 111, 203-305

Oscarson P, Ingemarson B, Larsson CM (1989) Growth and nitrate uptake properties of plants grown at different relative rates of nitrogen supply. II. Activity and affinity of the nitrate uptake system in Pisum and Lemna in relation to availability and nitrogen demand. Plant Cell Environ 12, 787-794

Palmer SJ, Berridge DM, McDonald AJS, Davies WJ (1996) Control of leaf expansion sunflower (Helianthus annus $\mathrm{L}$ ) by nitrogen nutrition. $J$ Exp Bot 47, 359-368
Pontallier JY (1990) A cheap quantum sensor using a gallium arsenide photodiode. Functional Ecology 4, 591-596

Radin JW, Boyer J (1982) Control of leaf expansion by nitrogen nutrition in sunflower plants, role of hydraulic conductivity and turgor. Plant Physiol 69, 771775

Radin JW, Parker L (1979) Water relations of cotton plants under nitrogen deficiency. I. Dependence upon leaf structure. Plant Physiol 64, 495-498

Reinink K, Blom-Zandstra M (1989) The relation between cell size, ploidy level and nitrate concentration in lettuce. Physiol Plant 76, 575-580

Reinink K, Groenwold R, Bootsma A (1987) Genotypical differences in nitrate content in Lactuca sativa $L$ and related species and correlation with dry matter content. Euphytica 36, 11-18

Richard JG, Sundstrom FJ, Grimes JA, Geaghan JP, Etzel WW (1985) Predicted effect of temperature and $\mathrm{N}$ fertilisation on crop response of four cultivars of head lettuce. Soil Sci Plant Anal 16, 583-613

Richardson S, Hardgrave M (1992) Effect of temperature, carbon dioxide enrichment, nitrogen form and rate of nitrogen fertiliser on the yield and nitrate content of two varieties of glasshouse lettuce. $J \mathrm{Sci}$ Food Agric 59, 345-349

Roorda Van Eysinga JPNL, Van Der Meijs MQ (1985) Effects of nitrogen nutrition and global radiation on yield and nitrate content of lettuce grown under glass. Soil Sci Plant Anal 16, 1293-1300

Schulze ED, Koch G, Percival F, Mooney HA, Chu C (1985) The nitrogen balance of Raphanus sativus $X$ raphanistum plants. I. Daily nitrogen use under high nitrate supply. Plant Cell Environ 8, 713-720

Van Der Boon J, Pieters JH, Slangen JHG, Titulaer $\mathrm{HHH}$ (1986) The effect of nitrogen fertilization on nitrate accumulation and yield of some field vegetables. In : Fundamental, Ecological and Agricultural Aspects of Nitrogen Metabolism in Higher Plants $(\mathrm{H}$ Lambers, JJ Neeteson, I Stulen, eds), Martinius Nijhoff, Boston, États-Unis, 489-492

Vermeulen J, Delvaux J, Vlassak K (1987) Effet de la fumure azotée et des conditions de croissance sur la teneur en nitrate des légumes à feuilles. Rev Agric 40, 879-893

Venus JC, Causton DR (1979) Plant growth analysis: a re-examination of the methods of calculation of relative growth and net assimilation rates without using fitted functions. Ann Bot 43, 633-638 